\title{
CONOCIMIENTOS QUE INTERACCIONAN EN LA ENSENANZA DE LAS CIENCIAS
}

\author{
MARÍN MARTÍNEZ, NICOLÁS \\ Departamento de Didáctica de la Matemática y de las Ciencias Experimentales. Universidad de Almería \\ nmarin@ual.es
}

\begin{abstract}
Resumen. En la enseñanza de las ciencias confluyen una diversidad de conocimientos: cotidiano, de ciencias, del alumno, académico o del profesor. Ante esto, cuando se fundamenta cualquier propuesta para enseñar ciencias se dan dos tendencias básicas según se enfaticen las analogías o las diferencias de los conocimientos citados. Este trabajo viene a sumarse a los que esgrimen las diferencias entre el conocimiento de ciencias y el del alumno para revisar propuestas didácticas que se basan en la analogía del «alumno como científico». Palabras clave. Analogía de «el alumno como científico», diferencias entre conocimiento de ciencias y del alumno, revisión crítica de modelos de enseñanza.

Summary. In Science Teaching different types of knowledge interact: the everyday knowledge of science of the student and the more academic knowledge of the teacher. Given this situation, any undertaking to teach science will show two basic tendencies, depending on whether the similarities or the differences between the two above-mentioned types of knowledge are emphasised. This paper is a further contribution to those that investigate this difference between the knowledge of teacher and student when looking at teaching proposals based on the analogy of student and scientist.

Keywords. Pupil as scientist, difference between the knowledge of teacher and student, teaching models.
\end{abstract}

\section{INTRODUCCIÓN}

Desde una perspectiva amplia, la enseñanza de las ciencias viene a significar una encrucijada cognitiva donde se dan cita una diversidad de conocimientos: académico, cotidiano, de ciencias, del alumno o del profesor.

En el ámbito de investigación de la didáctica de las ciencias han existido diferentes posiciones frente a la diversidad cognitiva, que básicamente se podrían reducir a dos:

- La tendencia más difundida percibe importantes analogías entre los conocimientos citados anteriormente. En concreto, los modelos para la enseñanza de las ciencias de mayor difusión se han fundamentado en alguna semejanza entre el conocimiento de ciencias y el del alumno. Muy extendido, este modo de proceder se ha denominado analogía o metáfora de «el alumno como científico»
(Solomon, 1994; Claxton, 1994; Marín, Solano y Jiménez Gómez, 1999; Yang, 1999).

Menos usual pero también relevante en el ámbito, existe otro modo de argumentar que resalta las diferencias entre el conocimiento de ciencias (y del experto de ciencias) y el del alumno, para hacer notar que las analogías entre ambos disminuyen al profundizar en el tema. Por tanto, sería conveniente revisar los modelos para la enseñanza de las ciencias que parten de éstas (Reif y Larkin, 1991; Pozo, 1999).

En esta última tendencia se enmarca este trabajo que pretende realizar una exhaustiva comparación entre el conocimiento de ciencias y el del alumno y utilizar las diferencias encontradas para revisar los modelos basados en la 
metáfora el alumno como científico. Previamente se establece un método para realizar las comparaciones cognitivas.

\section{ANALOGÍAS ENTRE EL CONOCIMIENTO DEL EXPERTO EN CIENCIAS Y EL DEL ALUMNO}

Si las posiciones epistemológicas más extremas del empirismo y del racionalismo mostraban grandes diferencias entre los conocimientos implicados en la enseñanza de las ciencias y, en particular, entre el conocimiento del experto de ciencias y el del alumno, las posiciones más moderadas del constructivismo han dejado entrever importantes semejanzas funcionales.

La metáfora del hombre de la calle como cientifico resalta las analogías que presentan las teorías personales usadas en el entorno cotidiano con las teorías del experto de ciencias (Kelly, 1955). Muchas de estas semejanzas se han mantenido en otros contextos teóricos y utilizando otros términos para referirse a las teorías personales tales como teorías implícitas (Pozo et al., 1991; Rodrigo et al., 1993; Oliva, 1999), teorías marco (Vosniadou, 1994), teorías de dominio (Karmiloff-Smith, 1994), teorías intuitivas (Carey y Spelke, 1994), esquemas cognitivos (Rumelhart y Ortony, 1982), esquemas alternativos (Driver, 1986), modelos mentales (Greca y Moreira, 1998), etc. Las principales analogías que se mantienen entre las teorías personales, y las del experto en ciencias han sido las siguientes:

- Los procesos psicológicos por los que se atribuyen o se asignan significados a una palabra, una frase, un dato empírico, una imagen, una percepción, etc. $\mathrm{y}$, en general, a cualquier producto de la interacción sujeto-medio son semejantes en el experto de ciencia y en el hombre de la calle. Ambos no son receptores pasivos de información, sino que utilizan sus teorías como marcos activos para interpretar la realidad (Piaget, 1977; Driver, 1986).

- Cada teoría hace referencia a un sector específico de la realidad de modo que el sujeto hace uso de ella para dar significados y abordar los problemas concretos que le plantea ese sector. En esta reiterada confrontación, la teoría se nutre y se desarrolla (Claxton, 1987).

- Las teorías guían y planifican la acción y, en concreto, la experimentación sobre el medio (Rodrigo et al., 1993). La actuación sobre el medio depende de las teorías que tengamos de él, pero lo que sucede depende de cómo es el mundo no de como creemos que es (Claxton, 1987).

- Las teorías personales y científicas son organizaciones cognitivas coherentes (Rodrigo et al., 1993; Oliva, 1996; Pozo, 1999). La coherencia de las primeras se basa en premisas diferentes a las segundas, lo que no significa que sean erróneas (Driver, 1986).

- Las teorías presentan una dinámica de confrontación semejante. Desde un punto de vista estructural, las teorías se pueden concebir como compuestas por un núcleo duro irrefutable y un cinturón protector de proposiciones hipotéticas que dirigen la experimentación personal en un intento de incrementar la capacidad predictiva del sistema y su coherencia interna sin poner en peligro sus construcciones nucleares (Carey, 1991; Luffiego et al., 1994).

Botella (1994) señala que la analogía del hombre de la calle como cientifico, en su versión no literal, ha resultado ser muy fructífera en estudios sobre personalidad, psicoterapia cognitiva, sobre el desarrollo del ciclo vital, en ciencia cognitiva, en filosofía de la ciencia o en psicología de la educación.

También la metáfora el alumno como científico constituye la premisa de los modelos y propuestas didácticas que han gozado de mayor aceptación en el ámbito de la educación en ciencias (Solomon, 1994; Claxton, 1994; Yang, 1999). Partiendo de diferentes aspectos de la analogía alumno como científico, los tres modelos de enseñanza más difundidos han sido:

- El primero de ellos, denominado por algunos autores movimiento de las concepciones alternativas (Gilbert y Swift, 1985), sigue las analogías cognitivas entre ideas del alumno y teorías del científico, de modo que, para diseñar la enseñanza, «lo que sabe el alumno» del contenido académico de ciencias es una pieza clave.

- El modelo de cambio conceptual percibe semejanzas notables entre los conflictos cognitivos que se dan en el progreso de las teorías de ciencias y los que se deben dar en el alumno cuando sus ideas, fuertemente arraigadas, interaccionan con las que les enseña el profesor.

- El modelo de enseñanza por investigación prevé mejoras sustanciales en el aprendizaje del alumno si en las clases de ciencias se simula la actividad de los grupos de investigación (Duschl y Gitomer, 1991; Gil, 1993).

\section{CONOCIMIENTOS IMPLICADOS EN LA EN- SEÑANZA DE LAS CIENCIAS}

Para que el acto de enseñar se pueda dar, debe estar presente el núcleo bipolar «algo a enseñar - alguien que lo ha de aprender». La ausencia de uno de los dos polos impide dicho acto; no así la ausencia de otros factores como el docente, el libro de texto, el escenario escolar o los compañeros de clase.

Existen discrepancias sobre qué términos usar para referirse al conocimiento que es enseñado al alumno en contextos escolares; unos autores prefieren denominarlo conocimiento escolar y otros, académico. Lo importante no será el término a usar sino mantener una cuidadosa distinción entre el conocimiento que va a ser enseñado (externo al aprendiz) y el conocimiento que finalmente terminará construyendo el alumno a partir de sus ideas previas. Se denominará al primero conocimiento académico a enseñar, y al segundo, conocimiento académico del alumno.

En el ámbito escolar, el profesor es la figura que normalmente media entre alumno y contenido objeto de enseñan- 
za (Artigue y Douady, 1986). Sin embargo, el «contenido» que finalmente llega al alumno de mano del docente dependerá del grado de comprensión de éste, de sus creencias sobre la enseñanza, de cómo percibe la construcción del conocimiento de las ciencias y el del alumno y, consecuentemente, del modo de enseñarlo. Los anteriores «efectos», aunque importantes, aquí no serán objeto de atención, por lo que el saber del docente se va a representar genéricamente por el conocimiento académico a enseñar.

Así pues, los dos tipos de conocimiento que se identifican en primer lugar en la enseñanza y el aprendizaje de las ciencias son: el conocimiento académico a enseñar y el conocimiento académico del alumno. Ambos constituyen el núcleo del escenario académico donde intervienen otros conocimientos (Rodrigo, 1997), tales como:

- El conocimiento de ciencias. Mediante algún tipo de transformación de este conocimiento, que se ha dado en denominar transposición didáctica (Jiménez Aleixandre y Sanmartí, 1997), éste se acondiciona para ser administrado en los distintos niveles educativos. Es decir, el conocimiento de ciencias da soporte de «autenticidad» al que hemos denominado conocimiento académico a ense- ñar. Así definido, se puntualiza que el conocimiento de ciencias a considerar es aquél que goza de un amplio consenso entre las comunidades de ciencias y es, en cierta medida, lo que Kuhn (1975) denomina ciencia normal.

- El conocimiento cotidiano del alumno. Éste es construido en una constante y dilatada interacción con su medio natural y social. El conocimiento cotidiano se adquiere usualmente de forma espontánea e informal, mientras que el académico se adquiere de forma más directiva, sistemática y formal. Salvo solapamientos tales como lo que se adquiere por currículo oculto o documentales de televisión, se admite que el conocimiento académico es claramente diferente al cotidiano (Rodrigo et al., 1993; De Posada, 1996; Pozo y Gómez Crespo, 1998). Ambos conforman el bagaje cognoscitivo que se denominará conocimiento del alumno.

- El conocimiento del científico o experto que realiza aportaciones al cuerpo de conocimientos de ciencias. Ubicado entre el conocimiento del alumno y el de ciencias, será esencial para una mejor comparación de estos últimos.

El esquema 1 se ha elaborado enfrentando conocimientos internos del sujeto (cotidiano y académico asimilado) con

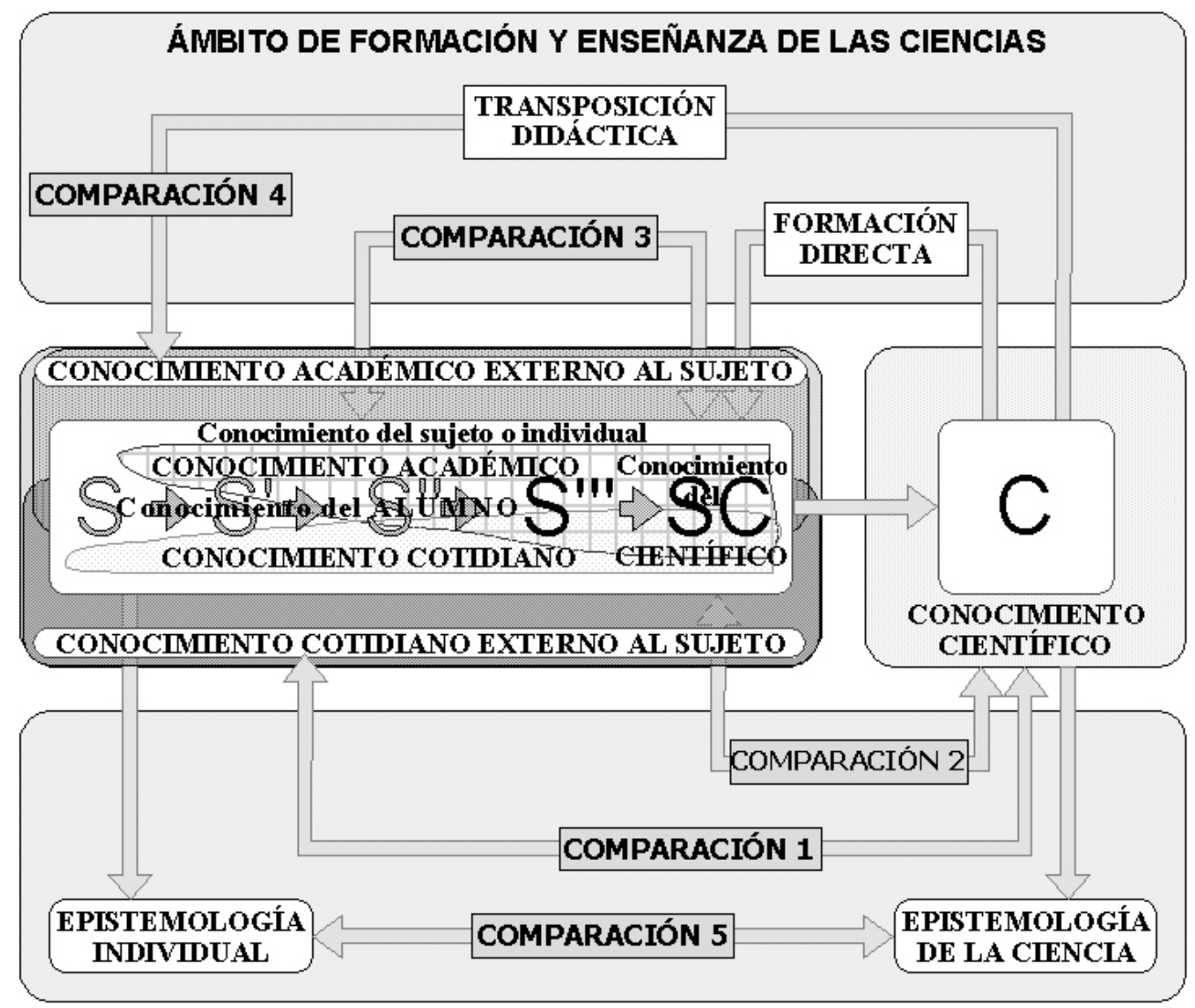


conocimientos externos (ciencias, cotidiano y académico externo). Tal distinción está bastante consensuada entre autores que han abordado problemas epistemológicos, por ejemplo, Lakatos (1974), Bunge (1981), Piaget y García, (1982), Chalmers (1984).

El proyecto de comparaciones cognitivas admite un buen número de posibilidades; ahora bien, centrándonos en las que podrían aportar argumentos para analizar o evaluar cuestiones problemáticas ligadas a la enseñanza y al aprendizaje de las ciencias, las comparaciones se reducen. A modo ilustrativo, proponemos brevemente cinco de ellas, así como la problemática que permitirían afrontar:

1) Comparación entre el conocimiento cotidiano y el de ciencias. Ambos conocimientos conllevan un proceso de construcción colectiva, pero sus compromisos sociales, finalidades de construcción y axiología son bien diferentes. La importancia de esta primera comparación cognitiva reside en que permite contextualizar otras, ya que se habla de los dos escenarios más relevantes, a falta del académico, donde se desarrollan los dos conocimientos individuales considerados: el conocimiento del alumno y el del experto en ciencias. Concretamente, con frecuencia, el conocimiento que el alumno desarrolla en su entorno cotidiano, lo utiliza para comprender las explicaciones del profesor, si es que el contenido de enseñanza queda cerca de su área de alcance cognitivo.

2) Comparación entre el conocimiento de ciencias y el del científico que es experto en algún sector disciplinar de las ciencias. Interesa resaltar esta diferencia para mostrar que, a pesar de ser dos conocimientos próximos, presentan mecánicas de construcción diferentes. Así, en la fase de construcción del conocimiento de ciencias donde interviene la comunidad de expertos, se dan procesos de regulación y administración que no aparecen en la actividad de los científicos o grupos de ellos (Kuhn, 1975). Por otro lado, esta actividad no es la misma si se refiere a la fase de descubrimiento (Chalmers, 1984) o tal y como se presenta en proyectos de investigación o en la fase de comunicación (Holton, 1972). Se han realizado propuestas para la enseñanza de las ciencias donde ambos conocimientos no parece que se hayan diferenciado suficientemente.

3) Comparación entre el conocimiento del experto en ciencias y el del alumno. Quizá porque se comparan conocimientos individuales, no es casualidad que los trabajos que han abordado esta confrontación cognitiva, se deba a autores del ámbito de la psicología (Schnotz et al., 1999). Desde un punto de vista funcional, esta comparación es semejante a la que se suele encontrar bien documentada y formulada como diferencias cognitivas entre expertos y novatos (Carey, 1991; Chi, Slotta y Leeuw, 1994). Aun siendo de otro ámbito, esta comparación puede aportar importantes sugerencias para la enseñanza de las ciencias si se tiene en cuenta que uno de sus objetivos más relevantes es desarrollar en el alumno una organización cognitiva y habilidades propias del pensamiento del experto de ciencias (Inhelder y Piaget, 1972; Shayer y Adey, 1984; Lawson, 1994). Además, cuanto más ajustados y precisos se hagan los diseños de enseñanza a los procesos de aprendizaje y desarrollo cognitivo que llevan al novato a convertirse en experto en ciencias, mayores serán las posibilidades para provocar esta transformación cognitiva.

4) Comparación entre el conocimiento de ciencias y el académico. Esta comparación es interesante en tanto aborda el problema de acondicionar el conocimiento de ciencias para su administración en el entorno escolar y analiza las diferencias entre conocimiento académico externo e interno al alumno. A la vez, es delicada de establecer, dado que sólo es posible estudiarla sobre discutibles vínculos causales entre modelos de transposición didáctica y modelos de enseñanza, y entre éstos y las adquisiciones del aprendiz. En efecto, tanto el conocimiento académico de ciencias a enseñar como el adquirido por el alumno en este ámbito pueden variar notablemente según la posición adoptada frente a diversas cuestiones (Osborne y Simon, 1996; Jiménez Aleixandre y Sanmartí, 1997; Pozo y Gómez Crespo, 1998). No obstante, en la literatura del ámbito se ha llegado a un consenso, más teórico que real, en el que se asumen ciertos vínculos causales en modelos tipificados como tradicionales o constructivistas (Jiménez Aleixandre, 2000). Este consenso haría posible documentar adecuadamente esta comparación.

5) Comparación entre modelos de construcción cognitiva individual y la construcción social del conocimiento de ciencias. La relevancia de esta comparación reside en el alto número de modelos didácticos realizados en el ámbito basados en supuestas semejanzas entre ambos tipos de construcciones: lo que se ha dado en denominar analogía del alumno como científico (Posner et al., 1982; Driver, 1983; Solomon, 1994; Claxton, 1994; Duit, 1999). Las posibles diferencias entre las construcciones cognitivas del alumno y las de ciencias podrían poner restricciones a los traspasos usuales del plano de ciencias al aula.

El desarrollo de este proyecto de comparaciones cognitivas iniciado al calor de los debates habidos en 1999 en la revista Enseñanza de las Ciencias terminó con una extensión excesiva. Esto obligó finalmente a reducir la exposición de comparaciones cognitivas a sólo una, entre el conocimiento de ciencias y el del alumno que, creemos, sintetiza y combina las mejores características de las citadas cinco comparaciones y aporta argumentos suficientes para continuar con el debate.

\section{CÓMO LLEVAR A CABO LA COMPARACIÓN COGNITIVA}

Para comparar con cierta sistemática el conocimiento de ciencias con el del alumno, se van a considerar las siguientes cuestiones:

A. ¿Cuál es el soporte físico del conocimiento? Se trata de señalar el soporte material donde está registrado el conocimiento. Subyace en este planteamiento simple una actitud epistemológica que se aleja de visiones que admiten un conocimiento «flotando» en un espacio etéreo esperando dueño. El constructivismo dinámico (Pozo, 1989; Delval, 1997; Marín y Benarroch, 2000) mantiene que el soporte del conocimiento individual es el propio sujeto. 
Aunque la influencia del conocimiento social en sus construcciones cognitivas sea enorme, éstas son siempre individuales.

Los conocimientos compartidos por un colectivo precisan de registros externos al sujeto (libros, revistas, soporte informático, etc.) mediante cualquier tipo de sistema de significantes.

B. ¿Cuál es el contexto donde se construye el conocimiento? Es importante considerar los condicionantes, compromisos, reglas, intereses, fines y valores que configuran el escenario o contexto en el que surge cada conocimiento. Estos factores son determinantes para facilitar después la caracterización del conocimiento construido (Rodrigo et al., 1993; Rodrigo, 1997). Los escenarios a considerar son el académico, el cotidiano y el científico.

C. ¿Cuáles son los modos de adquisición y construcción cognitiva? Mientras que en las construcciones cognitivas externas al sujeto es determinante la regulación y la difusión de las aportaciones individuales, en la construcción del conocimiento personal se admiten tres tipos de interacciones sujeto-medio a partir de las cuales el sujeto construye (Claxton, 1987; Rodrigo et al., 1993; Marín, 1997):

- Interacciones físicas o experiencias directas, que serían las que el sujeto lleva a cabo con los objetos materiales y personas que le rodean, como puede ser sujetar, transportar, cortar, transformar, construir, etc).

- Interacciones por significantes o experiencias simbólicas, donde el sujeto se apropia de buena parte del extenso bagaje cultural a través de significantes verbales, simbólicos, gráficos, etc.

- Experiencias vicarias o por observación, en las que el sujeto adquiere una valiosa información a través de la observación de las conductas de los demás, modelando la suya propia (Rivière, 1990).

La integración de datos provenientes de cada tipo de interacción conlleva una mecánica de aprendizaje específica (Claxton, 1987). Así, en las construcciones conceptuales es usual distinguir reestructuraciones débiles y fuertes. Las primeras suponen generalización de la extensión de los conceptos, diferenciación que conlleva la aparición de nuevos conceptos o la creación de nuevas relaciones entre ellos, sin que por ello se modifique el núcleo de conceptos de la teoría donde están insertos. Cuando el núcleo de la teoría cambia (reestructuración fuerte), también cambian los compromisos ontológicos del sujeto así como los significados de los conceptos que la estructuran (Carey, 1991). La acumulación de procesos de reestructuración débil va creando las condiciones necesarias para que se dé la fuerte (Pozo, 1989). Ésta se da más espaciada en el tiempo, es más difícil de provocar en la enseñanza y muchas de las reestructuraciones fuertes que se dan en el contexto científico no se llegan a dar en buena parte de la población (Shayer y Adey, 1984; Pozo y Gómez Crespo, 1998). Las construcciones cognitivas desde las interacciones que no son conceptuales, resulta más adecuado explicarlas me- diante diferentes procesos de equilibración, donde la unidad de organización cognitiva ya no sería el concepto sino el esquema cognitivo (Piaget, 1978).

En el caso de las construcciones sociales del conocimiento de ciencias existe una abundante literatura para explicar el progreso de teorías. Recientemente las nociones de reestructuración débil y fuerte se han usado con acierto para describir los diferentes cambios cognitivos en la historia de la ciencia (Thagard, 1992).

D. ¿Qué características presenta el conocimiento construido? Ésta será la cuestión más relevante para realizar la comparación cognitiva. Las dimensiones, caracteres o cualidades del conocimiento que se van a comparar son:

- Carácter implícito/explícito del conocimiento. El conocimiento externo al sujeto, para ser algo más que el intercambio oral de conocimientos individuales, está abocado a ser registrado en diversos formatos físicos, siendo más usual el escrito. Por el contrario, el conocimiento individual posee una importante carga de contenido cognitivo implícito. A pesar de su carácter implícito, es necesario considerar la presencia de este conocimiento para entender nuevas adquisiciones cognitivas o determinadas dificultades de aprendizaje, tanto en el ámbito cotidiano como académico (Piaget, 1976; Pozo et al., 1991; KarmiloffSmith, 1994; Rodrigo y Correa, 1999; Vosniadou, 1999).

- Contenido procedimental/declarativo del conocimiento. Es declarativo el conocimiento que se puede expresar mediante significantes y procedimental, el conocimiento que permite actuar sobre la realidad, procesar información tanto simbólica como empírica y aplicar el conocimiento declarativo para realizar inferencias, clasificar, prever, solucionar problemas y evaluar sus consecuencias (Pozo y Postigo, 1994; Lawson, 1994; De Posada, 1996). Si nos referimos al conocimiento del sujeto, lo declarativo y procedimental es un asunto difícil de precisar, pues depende del modelo cognitivo usado para interpretar al sujeto.

- Modos de asignar significados ligados a los significantes. En los conocimientos internos al sujeto, los significantes «juegan un papel de soporte de la estructura cognoscitiva y son instrumentos del pensamiento figurativo» (Piaget, 1980, p. 44), entre los que caben destacar los signos, símbolos, imágenes mentales y significantes verbales. Los significados son asignaciones o atribuciones que hace el sujeto a los significantes, usando para ello su estructura cognoscitiva (Inhelder y Piaget, 1972), la cual ha ido construyendo desde sus interacciones con el medio. Así, usando la anterior distinción se puede afirmar que en las adquisiciones académicas del alumno, en contextos de enseñanza usuales, el significado que asocia a los conceptos aprendidos se aproxima bastante a su versión escrita, y su carácter, un tanto rígido, imposibilita o dificulta la transferencia cognitiva (Pozo y Gómez Crespo, 1998). Por el contrario, los significados que usualmente asigna el alumno a conceptos propios del escenario cotidiano, al construirse desde una diversidad de interacciones (físicas, simbólicas y vicarias) en períodos de tiempo amplios, poseen una carga procedimental importante en la que lo cognitivo y lo afectivo está entremezclado. 
En los conocimientos externos al sujeto, como el de ciencias, un concepto toma su significado por su relación con otros conceptos del entramado conceptual en el que está inserto y por su uso en los distintos contextos sociales. Se denomina asimilación recíproca al proceso de regulación social por el que el intercambio conceptual va conformando un significado consensuado que permite la comunicación interpersonal, y cuyo margen de tolerancia, en el caso de la ciencia, se procura reducir al mínimo (Marina, 1998; Di Sessa y Sherin, 1998).

\section{COMPARACIÓN ENTRE EL CONOCIMIEN- TO DE CIENCIAS Y EL DEL ALUMNO}

Las analogías entre el conocimiento de ciencias y el del alumno se perciben superficiales cuando se profundiza en los detalles sobre como son construidos uno y otro (Claxton, 1994). Con cierta intención sistemática, se puede caracterizar estas diferencias en cuatro aspectos relevantes:

\section{Soporte fisico}

Mientras el soporte del conocimiento del alumno es su propia mente, en el de ciencias existe una diversidad de formatos y soportes externos (libros, revistas especializadas, actas de congresos, soportes informáticos, etc.) donde lo usual es registrarlos con significantes verbales, matemáticos y gráficos. Por tanto, las construcciones de las teorías personales son individuales, aunque en éstas sean determinantes sus interacciones sociales; y las construcciones de las teorías de ciencias son primordialmente de índole social aunque se utilicen como material básico las aportaciones individuales.

\section{Contexto donde se construye el conocimiento}

Existen tres escenarios donde se desarrollan ambos conocimientos de manera desigual:

a) El escenario cotidiano acoge una diversidad de colectivos y tipologías individuales. Usualmente, lo que es objeto de ser tratado como problema es más bien el obstáculo que impide conseguir los objetivos de la actividad cotidiana (laboral, lúdica, de relación social, etc.). Las soluciones son usualmente pragmáticas, particulares y no requieren ser útiles para otros problemas u otras personas. Los valores de este escenario están muy diversificados según grupos sociales y tipos de actividad.

b) El escenario académico, donde se transmite a los alumnos una serie de conocimientos estructurados y parcializados en disciplinas. Los fines y valores de este escenario dependen del modelo educativo, aunque es habitual que exista un desfase entre la práctica y los fines educativos declarados. Estos últimos casi siempre hacen referencia a la educación integral del alumno, igualdad de oportunidades, transferencia del conocimiento adquirido en clase para una mejor comprensión y actuación en el entorno cotidiano actual, etc. Algunos de estos objetivos se logran en parte, pero la meta real que predomina es la propedéutica: superar exámenes para pasar al siguiente nivel académico.

c) El escenario de ciencias, donde se construye este conocimiento muestra peculiares mecanismos para que la producción cognitiva sea convergente y coherente. Los fines y valores predominantes están mediatizados por el esfuerzo en producir un conocimiento válido, fiable y eficaz, principalmente para su uso pragmático en el ámbito de producción de bienes materiales (Vázquez et al., 2001), que es donde este conocimiento mantiene su mayor compromiso (Chalmers, 1984). Como en otros conocimientos, no hay límites para hacer construcciones cognitivas, pero la ciencia se distingue por el segmento material (físiconatural) de la realidad con el que interactúa y por la dinámica de confrontación constante e interpersonal. En este segmento se afrontan y se buscan soluciones a una gran variedad de problemas que a la vez generan otros nuevos. Las soluciones al problema, siempre provisionales, deben mostrarse útiles y consensuadas.

El escenario de ciencias descrito determina fuertemente el conocimiento de ciencias construido, pero no hay que olvidar las complejas relaciones CTS (Fernández et al., 2002) que también lo vinculan a la parte más interesada y material del escenario cotidiano, hasta el punto que determinan un buen número de direcciones de investigación (problemas medioambientales, desarrollo tecnológico, producción y control de bienes, etc.). Por el contrario, el alumno adquiere desde la diversidad de actividades del escenario cotidiano (cognitivas, afectivas y motrices) un conocimiento eficaz para los problemas diarios pero corto para el escenario académico.

\section{Modos de adquisición y de construcción cognitiva}

A. El alumno construye su conocimiento a través de tres tipos de interacciones básicas: físicas, vicarias y simbólicas (Rodrigo et al., 1993; Marín, 1997). En el alumno de la etapa infantil son más relevantes las interacciones físicas que dan lugar a una estructura cognitiva de carácter procedimental e implícita muy ligada a lo afectivo. Después, en el proceso de socialización, comienzan paulatinamente a ser más relevantes, sus interacciones simbólicas, que le permiten construir sobre las anteriores otras estructuras de carácter conceptual. Esta doble estructuración cognitiva (conceptual y semántico-vivencial) -y sus vínculos- permite explicar mejor la asignación de significados individuales (Claxton, 1987; Pozo, 1989).

Así pues, en el ámbito cotidiano, el alumno construye un conocimiento que posee un importante componente individual de carácter procedimental e implícita donde lo cognitivo y lo afectivo están fuertemente ligados. Este conocimiento queda lejos del alto grado de estructuración lógica del entramado conceptual del conocimiento de ciencias, pero es pragmático, adaptativo, fuertemente arraigado y, aunque distante del científico, útil y eficaz en el ámbito cotidiano (Pozo, 1999). Sin embargo, el mismo conocimiento es torpe y limitado en el entorno académico, que se manifiesta excesivamente local, egocéntrico y global (MEC, 1989). 
En el ámbito académico, el alumno adquiere en períodos de tiempo relativamente cortos gran cantidad de contenidos, normalmente en su versión declarativa, por lo que construye un conocimiento pobre en procedimientos y con poca flexibilidad para ser transferido. Alcanza sus mejores logros en el artificial contexto académico pero tampoco habría que desdeñar su utilidad en entornos cotidianos, ya que el nuevo léxico adquirido por el alumno le abre nuevas vías de comunicación y entendimiento; incluso, le aporta cierta formación procedimental (pensamiento matemático, habilidades lectoras, estructuración categorial), abundantes adquisiciones declarativas más memorísticas que significativas y con poca relación con el conocimiento cotidiano (Ausubel et al., 1986; De Posada, 1996) y, ocasionalmente, algunas habilidades procedimentales científicas (Shayer y Adey, 1984; Marín 1986; Pozo y Gómez Crespo, 1998).

La mayor parte de las construcciones cognitivas en contextos cotidianos y académicos pueden ser explicadas por procesos cognitivos de memorización, generalización y diferenciación (Pozo, 1989). Los desequilibrios cognitivos de mayor envergadura, si es que ocurren, se dan espaciados en el tiempo, por lo que pocos alumnos superan el nivel de operaciones concretas $y$, por tanto, quedan lejos de desarrollar un pensamiento semejante al del científico (Shayer y Adey, 1984). Así, lo usual es que el alumno utilice estrategias inductivas o de ensayo y error.

La regulación social del conocimiento del alumno actúa por igual sobre su sistema cognitivo y afectivo y se rige principalmente por pautas de inserción y pertenencia a diversos grupos de su entorno inmediato (familia, amigos, grupo escolar, etc.). Aunque la integración individual depende de la idiosincrasia de cada grupo, lo usual es que el entramado de normas, valores, creencias y vínculos afectivos del grupo sean monedas de intercambio de más valor que, por ejemplo, las cualidades manuales o intelectivas.

B. El proceso de construcción del conocimiento de ciencias presenta una fase de construcción individual, usualmente en el contexto de equipos de investigación, y otra fase de construcción social donde interviene de forma decisiva la comunidad de expertos. No cabe duda de que la construcción del conocimiento de ciencias es eminentemente social. La formación del investigador, su producción en el seno de grupos de investigación, la incorporación de las aportaciones individuales, las motivaciones e intereses para delimitar problemas, la aplicación del conocimiento de ciencias, etc. son procesos claramente sociales. Sin embargo, esto no es argumento suficiente para no considerar las diferencias entre la fase individual y colectiva.

En la fase individual, la producción del experto está en sintonía con su formación científica desde el cuerpo de conocimientos actuales. El proceso de formación se inicia en contextos académicos para continuar en el ámbito profesional, donde es determinante el contexto de recursos humanos y materiales, así como el esfuerzo personal. El científico no podrá evitar que sus creencias, intereses, problemas profesionales o personales, afiliaciones a grupos o ideas, conocimiento implícito y demás factores, no siem- pre «tan científicos», influyan tanto en su formación como, consecuentemente, en su producción (Holton, 1972).

En el quehacer diario del experto de ciencias es frecuente el incremento de conocimientos específicos por reestructuración débil mediante, por ejemplo, una lectura continuada, congresos, reuniones, etc., así como a través de experimentos y observaciones de laboratorio. Además, los anteriores escenarios y fuentes de información suponen un proceso continuo de perturbación cognitiva que lleva al experto a continuas reestructuraciones fuertes que dan oportunidad a desarrollar un potente pensamiento hipotético deductivo que lo generaliza a los más diversos contextos cognitivos.

En la fase colectiva, se podría pensar que, si el conocimiento de ciencias se nutre de las aportaciones individuales, éste heredará la subjetividad de cada científico. Eso en parte es cierto, pero se dan varios mecanismos de regulación social que hacen que lo que termina incorporándose al «cuerpo de conocimientos» sea un producto más elaborado, depurado, aséptico, racional y coherente. En efecto, por un lado, el científico se ve obligado a publicar sus aportaciones en su versión más racional y en el lenguaje consensuado de su comunidad; y, por otro, la incorporación e incidencia de las aportaciones individuales al cuerpo de conocimientos colectivos de ciencias están, con un rigor supuestamente racional, reguladas por una comunidad de científicos amplia (Holton, 1972). Así pues, el conocimiento de ciencias no es sólo la versión escrita del pensamiento del científico. Además, con el paso del tiempo, otros mecanismos de selección (Toulmin, 1972), de cambios de paradigmas (Kuhn, 1975) o programas de investigación (Lakatos, 1983), etc. intervienen para eliminar, decantar, enfatizar, ampliar, regular empíricamente, difundir selectivamente o formalizar la producción individual, sin descartar otras valoraciones externas asociadas a la utilidad del conocimiento producido (Chalmers, 1984).

Hay que notar que los factores de regulación social, mientras que en el alumno actúan como condicionantes externos, en la ciencia sólo actúan como externos en la fase de producción individual. En la fase de conocimiento socialmente construido, tanto en la mecánica que regula la incorporación de las aportaciones individuales al grueso de conocimiento consensuado de ciencias como en la mecánica de desarrollo y cambio de teorías, los factores sociales son directamente determinantes de las construcciones cognitivas.

\section{Carácter y diferencias del conocimiento construido}

Las diferencias más destacables entre el conocimiento de ciencias y el del alumno son las siguientes:

1) Diferencias sobre aspectos especificos del conocimiento vinculados a fenómenos físico-naturales. La ciencia, al ser un conocimiento socialmente compartido por una comunidad de expertos, requiere ser registrada en diferentes soportes de un modo declarativo mediante símbolos aceptados y consensuados por la comunidad (verbales, matemáticos, gráficos, etc.). A fin de evitar confusiones, se reduce al mínimo el margen de tolerancia del significado de 
los conceptos precisándolos al máximo mediante una organización conceptual con una coherencia interna alta y que en buena medida se puede asimilar a la lógica de clases (Pozo, 1989; Marina, 1998). La precisión y consenso conceptual se extrema, puesto que se espera de este conocimiento que sea útil y eficaz para resolver problemas del ámbito económico y social donde tiene adquiridos fuertes compromisos y responsabilidades.

Este esfuerzo por construir un conocimiento altamente coherente, además de actuar sobre el entramado conceptual, se procura mantener también entre las partes que integran el cuerpo de ciencias y entre éstas y las confrontaciones empíricas.

El conocimiento del alumno no se desarrolla en un contexto de tanta exigencia cognitiva, por lo que el grado de coherencia interna es menor que el de ciencias. Está orientado a ser un conocimiento funcional, pragmático y útil para las actividades cotidianas, en muchas de las cuales las cualidades afectivas cuentan más que las cognitivas. Esto hace que el conocimiento que posee el alumno de los fenómenos físico-naturales, comparado con el de ciencias, sea global, centrado en un entorno específico cotidiano, subjetivo, en buena parte implícito y vinculado a su sistema afectivo.

Las diferencias más relevantes entre los aspectos específicos del conocimiento de ciencias y del alumno son:

- Desde el punto de vista cualitativo: mientras el conocimiento de ciencias se expresa usualmente mediante un entramado conceptual altamente organizado y coherente, de modo que un concepto adquiere su significado por su posición y relación en la trama conceptual y se puede expresar a través de otros significantes, el conocimiento del alumno contiene una doble estructura cognitiva donde parte del entramado conceptual está fuertemente vinculado con el vivencial. Esto hace que en muchos contenidos cognitivos del alumno, lo conceptual, lo afectivo y lo sensomotriz esté fuertemente mezclado y que otros, contenidos implícitos, ni siquiera tienen vínculo con lo conceptual u otro tipo de significantes (Piaget, 1976; Kamirloff-Smith, 1994). En muchos casos, el significado que asigna el sujeto a un concepto viene dado por una serie de vivencias orgánicas, cognitivas, afectivas y motrices (Castilla del Pino, 2000).

- Desde el punto de vista cuantitativo: existe buen número de contenidos cognitivos del alumno que no admiten vínculos lógicos o correspondencias con ningún contenido académico de ciencias y, lo contrario, el repertorio de esquemas cognitivos del alumno sólo da significado más o menos adecuado a unos cuantos contenidos académicos; el resto los desconoce. Esto es un detalle que parece haberse pasado por alto en un buen número de trabajos donde al alumno se le han asignado «concepciones» que sólo estaban en la mente del investigador (Marín, Solano y Jiménez Gómez, 2001).

2) Diferencias sobre aspectos generales del conocimiento. Lo usual es que el alumno afronte las tareas y problemas cotidianos con estrategias inductivas o por «ensayo y error». El modo de procesar, el alumno, la información externa está muy determinado por lo concreto y lo perceptivo; percibe como necesario explicar lo que cambia, no lo que permanece. Establece relaciones con limitaciones tales como enfatizar una dirección preferente en interacciones no lineales o establecer relaciones causales de semejanza y contigüidad (Driver, 1986; Pozo y Gómez Crespo, 1998). El entorno académico, más preocupado por enseñar y evaluar la versión declarativa de los contenidos de ciencias, no potencia el desarrollo procedimental, de manera que son pocos los alumnos que alcanzan el nivel formal (Shayer y Adey, 1984). Las características procedimentales anteriormente señaladas permanecen en la mayoría de la población estudiantil.

En ciencias, los procedimientos varían según la fase constructiva del conocimiento en que centremos la atención. Las fases que conviene distinguir son las que siguen: a) fase previa en que los expertos, individualmente o en grupo, diseñan la investigación y el modo de abordar los problemas; $b$ ) fase de descubrimiento en que los científicos llevan los diseños a la acción; $c$ ) fase de comunicación; d) fase de regulación social; yf) fase de justificación.

Normalmente la fase previa de una investigación conlleva una planificación donde subyace una estructura hipotéticodeductiva (Bunge, 1981). En la fase de descubrimiento, aunque se procura mantener la línea de trabajo marcada, la búsqueda de soluciones se hace farragosa y tortuosa, sobre todo en zonas fronterizas en las que el conocimiento disponible se vuelve torpe. En éstas aparecen procesos de rectificación, de repetición, de nuevas experiencias que controlan mejor las variables, de ensayo y error, etc., procedimientos semejantes a los que usa el alumno. No hay que olvidar que al experto nunca le faltarán situaciones problemáticas donde se manifieste procedimentalmente como un novato. Después, en la fase de comunicación no se suelen reflejar todas las vivencias del investigador de la fase de descubrimiento. Los momentos de duda y zozobra en que se han usado procedimientos pocos científicos o se han mantenido ideas o sentimientos poco racionales se suelen obviar en la elaboración del informe de la investigación (Holton, 1972) de modo que entre el problema y la solución se traza una línea argumental racional y coherente.

En la fase de regulación social se incorporan y difunden las aportaciones individuales, lo que conlleva la ampliación del entramado conceptual del cuerpo de conocimientos, manteniendo la coherencia interna y el consenso. En esta fase, se dan un conjunto complejo de procedimientos donde un modelo organicista sería el más apropiado para entenderlo (Luffiego, 2001). De hecho, las nuevas aportaciones no se incorporan como simples añadidos o por acumulación sino mediante un proceso que bien se podría explicar por asimilación y acomodación (Piaget, 1974; Kuhn, 1975). Sobre el producto individual, se produce un nuevo proceso de reconstrucción racional eminentemente social (Lakatos, 1974; Chalmers, 1984). Del conocimiento así construido se ocupa la filosofía de las ciencias en la fase de justificación, cuya cuestión primordial es establecer pautas metodológicas que expliquen el progreso de las teorías de ciencias (Lakatos, 1974; Piaget y García, 1982; Chalmers, 1984). Aunque los modelos propuestos para explicar el progreso de las teorías de ciencias difieren no- 
tablemente entre sí (Piaget y García, 1982), en la actualidad son mejor aceptados los que son coherentes con la posición epistemológica que defiende el constructivismo, lejos de las posiciones positivistas y racionalistas más extremas (Izquierdo, 2000).

En pocas palabras, excepto en la fase de descubrimiento donde aparecen procedimientos semejantes a los del alumno, en las demás fases de la construcción del conocimiento de ciencias aparecen procedimientos hipotéticodeductivos que se dan escasamente en el entorno cotidiano e, incluso, en el académico (Fernández et al., 2002).

El esquema 2 ofrece una síntesis de las diferencias más destacadas entre el conocimiento del alumno y el de ciencias.

\section{REFLEXIONES SOBRE LA ENSEÑANZA DE LAS CIENCIAS}

Las diferencias cognitivas entre el conocimiento del alumno y el de ciencias permiten revisar los modelos para la ense- ñanza de las ciencias que se fundamentan en analogías cognitivas entre ambos conocimientos. Veámoslo:

1) El movimiento de las concepciones alternativas sugiere que uno de los factores relevantes para diseñar la enseñanza, si no el que más, es lo que sabe el alumno del contenido a enseñar (Driver, 1986). Esta propuesta para enseñar ciencias, incluso desde las diferencias encontradas entre el conocimiento de ciencias y el del alumno, es más que razonable: sólo son posibles salvarlas si, al enseñar ciencias, se tiene en cuenta el conocimiento del alumno. No obstante, las diferencias entre ambos conocimientos permiten formular una crítica a este modelo de enseñanza: «sus medidas didácticas son deficientes, pues se basan en información del alumno demasiado limitada y sesgada» (Marín, Solano y Jiménez Gómez, 2001).

La principal razón que sostiene la anterior afirmación se encuentra en las diferencias encontradas entre el conocimiento de ciencias y el del alumno: cuando el investigador diseña cuestionarios para indagar sobre lo que sabe el alumno e interpreta después sus respuestas, lo hace principal-

Esquema 2

Diferencias entre el conocimiento de ciencias y el del alumno.

\begin{tabular}{|c|c|}
\multicolumn{1}{|c|}{} & \multicolumn{1}{c|}{ Conocimiento del alumno } \\
\hline $\begin{array}{c}\text { Soporte } \\
\text { ¿extraño } \\
\text { o interno? }\end{array}$ & $\begin{array}{l}\text { El soporte es orgánico (la mente). } \\
\text { Las construcciones cognitivas son } \\
\text { individuales por mucha influencia que tengan } \\
\text { los factores sociales. }\end{array}$ \\
\hline $\begin{array}{c}\text { Contexto } \\
\text { donde se } \\
\text { desarrolla } \\
\text { el } \\
\text { conocimiento }\end{array}$ & $\begin{array}{l}\text { De la diversidad de actividades del escenario } \\
\text { cotidiano surge un conocimiento eficaz para } \\
\text { los problemas cotidianos pero corto en el } \\
\text { escenario académico. Ambos conocimientos } \\
\text { presentan pocos vínculos pues se adquieren } \\
\text { de modo diferente. }\end{array}$ \\
\hline \multicolumn{1}{|c|}{} \\
\hline
\end{tabular}

La construcción cognitiva es doble, conceptual y vivencial. La mayoría de las adquisiciones se explican por procesos de reestructuración débil. La fuente, si ocurre, se da muy espaciada, por lo que pocos alumnos superan el nivel concreto. La regulación social es externa.

- Aspectos específicos: parte de la estructura vivencial está ligada a la conceptual y parte es implícita. El significado es vivencia orgánica.

- Aspectos procedimentales. De tipo inductivo o ensayo y error. La información externa se procesa de forma muy limitada.

\section{Conocimiento de ciencias}

Soportes externos (revistas, libros, informática, etc.). Al ser compartido, se debe registrar a través de significantes (signos, gráficos, palabras...).

El escenario de ciencias tiene fuertes compromisos con ámbitos de producción de bienes que exige un conocimiento válido, eficaz y que dé soluciones a largo plazo. Fuerte compromiso cognitivo. Los vínculos CTS marcan la dirección de un buen número de investigaciones.

Fase individual: la confrontación en debates y laboratorio multiplican las reestructuraciones fuertes, de donde surge potente pensamiento formal.

Fase social: factores sociales (int.-ext.) regulan la incorporación individual, así como el desarrollo y aplicación de las ciencias.

Excepto en la fase de descubrimiento donde los tanteos del científico y del alumno se asemejan, en las demás fases de la construcción de ciencias (previa, comunicación, regulación social y justificación), predominan los procedimientos hipotético-deductivos. 
mente desde su formación en el conocimiento de ciencias. El sesgo y distorsión que comente el investigador será parecido al que tendría un análisis social de la época romana desde la contemporánea. Aunque siempre hay sesgo, al usar los esquemas cognitivos propios de su formación en ciencias, el investigador provoca un sesgo aún mayor.

El sesgo más relevante cuando se intenta perfilar el conocimiento del alumno desde el científico es que se recoge una información restrictiva. En efecto, o bien sólo se percibe parte del conocimiento declarativo del alumno relacionado con el académico o bien su desconocimiento del tema. Esta última opción, muy frecuente, es una consecuencia de diseñar cuestionarios con preguntas formuladas en un lenguaje académico y con un nivel de dificultad que dan poca oportunidad de expresión al conocimiento del alumno (Marín, Solano y Jiménez Gómez, 2001). Desde la visión de conocimiento de ciencias, no se percibe buena parte del bagaje cognitivo del alumno que ha sido construido desde sus vivencias cognitivas y afectivas en escenarios cotidianos, justo aquellas ideas y contenidos cognitivos implícitos de naturaleza procedimental distintos a los contenidos académicos.

En definitiva, desde la perspectiva del conocimiento de ciencias se toma una información limitada y sesgada. del alumno Las orientaciones didácticas que se deducen de esta información heredarán tales deficiencias.

2) El modelo de cambio conceptual parte del supuesto de que los conflictos cognitivos que se dan en el progreso de las teorías de ciencias tienen algunas similitudes con los que sufre el aprendiz de ciencias. Es posible que a cierto nivel de simplificación se pueda percibir cierta analogía, pero la comparación entre el conocimiento de ciencias y el del alumno muestra que las construcciones cognitivas de estos conocimientos son diferentes. Esto permite afirmar que el modelo de cambio conceptual ofrece una visión del aprendizaje parcial y errónea y su propuesta de enseñanza queda lejos de provocar el aprendizaje previsto (Marín, 1999).

Para el conocimiento de ciencias puede ser lógico hablar de cambio conceptual, pues es un tipo de construcción cognitiva interpersonal que precisa ser registrado usualmente mediante un entramado conceptual. Dicho entramado podría servir de modelo para las construcciones conceptuales del alumno pero no así para sus estructuras semántico-vivenciales, puesto que poseen bastante contenido de carácter implícito y procedimental (Piaget, 1974; Karmiloff-Smift, 1994), donde lo cognitivo y lo afectivo está fuertemente interrelacionado (Botella, 1994; Marina, 1998; Pintrich, 1999) y cuya mecánica constructiva es diferente a la conceptual (Claxton, 1987).

Por otro lado, la secuencia de enseñanza que propone el modelo de cambio conceptual consiste en presentar una serie de evidencias para generar en el alumno cierto conflicto cognitivo. Se trata de crearle cierta insatisfacción con sus ideas, para después presentar las nuevas como más plausibles y útiles. Esta secuencia es discutible, puesto que las evidencias que prepara el docente para crear conflictos cognitivos usualmente no actúan como tales en los alumnos (Villani y Orquiza de Carvalho, 1995; Leach, 1999).

Además, admitiendo que el sujeto afronte el conflicto como tal, existen varios modos para compensar la perturbación que, en cualquier caso, más que un cambio, supone modificaciones en los esquemas cognitivos. Puede ocurrir que: a) aumente en extensión la capacidad asimiladora de éstos; b) se coordinen entre ellos; c) se creen nuevos por diferenciación; o $d$ ) se creen otros de rango superior (Piaget, 1978). Estas posibilidades de construcción cognitiva, bien fundamentadas en datos psicológicos, no son contempladas en la propuesta de aprendizaje del cambio conceptual (Claxton, 1987; Pozo y Gómez Crespo, 1998; Oliva, 1999; Marín, 1999). Así, el supuesto cambio conceptual no sería posible, pues ni hay cambio ni, en muchos casos, es conceptual (Marín, 1999; Pozo, 1999). En la práctica, aún no se dispone de experiencias de clase, donde el conflicto cognitivo haya dado lugar a un claro cambio conceptual (Duit, 1999).

3) El modelo de enseñanza por investigación sugiere simular en clase la actividad de los grupos de investigación (Duschl y Gitomer, 1991; Gil, 1993) en el supuesto de que tales condiciones de enseñanza favorecen el aprendizaje. El primer problema que se encuentra este modelo es el de la simulación en clase de la actividad científica. Esto supone implicar al alumno en el desarrollo de actividades procedimentales (precisar problemas, controlar variables, formular y contrastar hipótesis, etc.) para los que no tiene las capacidades cognitivas adecuadas. En efecto, menos del $30 \%$ de los estudiantes de secundaria alcanzan algunas habilidades procedimentales usadas por los científicos (Shayer y Adey, 1984; Marín, 1986).

La simulación de la actividad de ciencias en clase podría ser factible si se consideran las diferencias procedimentales entre el conocimiento del alumno y el de ciencias. En concreto, el alumno no sólo presenta limitaciones procedimentales respecto a las de ciencias, sino que también posee otras relevantes capacidades operacionales que serán tanto más diferentes a las científicas cuanto más inferior sea el nivel cognitivo en el que se encuentre (Inhelder y Piaget, 1972; Piaget, 1977; Pozo y Gómez Crespo, 1998).

Además, creer que la enseñanza que simula la actividad científica mejora el aprendizaje de las ciencias (Valdés et al., 2002) supone asumir cierto isomorfismo entre la actividad de clase por investigación y la mecánica de aprendizaje del alumno. Es dudoso que los procesos constructivos del aprendiz sean similares a los que usan en la construcción de las ciencias, dadas las diferencias notables entre unos y otros (Pozo y Gómez Crespo, 1998).

La razón básica que soporta las anteriores críticas es común a los tres modelos revisados: la notable distancia entre conocimiento de ciencias y del alumno que hace ver desde lejos la organización cognitiva y el aprendizaje de éste. Un contexto teórico fundamentado en datos psicológicos lo vería más cerca y menos distorsionado. En esta misma dirección crítica, se podría revisar el concepto que se mantiene sobre aprendizaje, y sus vínculos con la enseñanza, que es común a todos los modelos basados en la 
analogía del alumno como científico (en lo sucesivo, modelos $A c C$ ):

Un primer argumento crítico afirma que los modelos $A c C$ prevén mayor prestancia del alumno para aprender que la que reflejan los modelos mejor fundamentados en datos psicológicos o lo percibido en el aula. Así, parece ser más difícil de lo previsto crear conflictos cognitivos (Villani y Orquiza de Carvalho, 1995; Duit, 1999), relacionar sustancialmente ideas previas y conceptos de ciencias (Pozo y Gómez Crespo, 1998) o adquirir habilidades procedimentales para desarrollar pequeñas investigaciones como lo hacen los científicos (Shayer y Adey, 1984; Lawson, 1993). En definitiva, desde los modelos $A c C$ se mantiene una visión optimista de los efectos que prevén sus diseños de enseñanza a pesar de la falta de confirmación de éstos.

Enseñar y aprender son dos procesos diferentes. Enseñar hace referencia a las condiciones y acciones docentes externas al sujeto, dirigidas a provocar algún tipo de modificación en su sistema cognitivo o afectivo, mientras que aprender se refiere a dicha modificación interior (Delval, 1997). Así, un exquisito diseño de enseñanza de ciencias no garantiza un buen aprendizaje. Factores internos del sujeto, como su nivel cognitivo, sus ideas previas o su estado emotivo, filtran, alteran o malogran el supuesto efecto lógico de tal intervención. Mientras el profesor propone, el alumno dispone.

Un segundo argumento crítico afirma que en los trabajos alineados a $A c C$ se percibe cierta indiferencia, quizá confusión, entre enseñar y aprender. Son numerosos los argumentos que vinculan directamente aprendizaje y actividad científica. He aquí algunos ejemplos:

- La propuesta de cambio conceptual inicial (Posner et al., 1982) se fundamenta en que existen pautas análogas de cambio conceptual en el aprendizaje con el propuesto por la filosofía de la ciencia contemporánea.

- La estrategia de enseñanza que nos parece más coherente con la orientación constructivista y con las características del razonamiento cientifico es la que plantea el aprendizaje como tratamiento de situaciones problemáticas abiertas [análogas a las que se dan en la actividad de los cientificos] que los alumnos puedan considerar de interés (Gil et al., 1999).

- Cudmani y otros (2000) hacen una propuesta fundamentada en la epistemología de la ciencia de Laudan para desarrollar «un modelo de aprendizaje integrador de aspectos conceptuales y no conceptuales de las ciencias».

- En un debate en la revista International Journal of Science Education sobre modelos de enseñanza y aprendizaje en ciencias (Gobert, 2000), se presentan algunos de ellos como «modelos de aprendizaje» o «modelos mentales» cuando en realidad son estructuras conceptuales de un tema de ciencias construidas alrededor de ideas claves que se supone que son necesarias para mejorar la comprensión del alumnado (Clement, 2000).
Desde la historia, la filosofía y la estructura disciplinar de las ciencias se pueden hacer valiosas propuestas de instrucción, intervención o enseñanza de ciencias, pero éstas no pueden llegar a considerarse propuestas de aprendizaje, dado que la fractura entre conocimientos individuales y compartidos lo impiden (Vosniadou, 1999; Clement, 2000). Argumentar sobre aprendizaje desde $A c C$ puede suponer mostrar creencias e intuiciones sobre aprendizaje poco adecuadas (Pozo y Scheuer, 1999).

Además, las aportaciones a la enseñanza de la ciencia no son exclusivas de los modelos $A c C$. Otros modos de construcciones cognitivas del aprendiz diferentes a los de ciencias pueden aportar sugerencias didácticas complementarias a las realizadas desde $A c C$. Así, por ejemplo:

- Lo que permite el desarrollo de un conocimiento flexible y transferible es la adquisición de significados con rica carga procedimental, y no tanto conocer el «significado» mediante una exposición verbal de un concepto (Pozo y Gómez Crespo, 1998; Vosniadou, 1999). La fragmentación del conocimiento que se produce al enfatizar la versión declarativa de los conceptos, tan usual en el ámbito académico, restringe seriamente la adquisición de significados por parte del alumno.

- La adquisición de significados es una cuestión de grados. Sobre cualquier entidad susceptible de ser cognoscible (un concepto, una relación, un fenómeno, etc.), el sujeto siempre dispone de cierta carga de significados que puede ser enriquecida constantemente, y esto es cierto tanto para el hombre de la calle como para el científico más eminente. No es, por tanto, una cuestión de todo o nada (Pozo, 1996; Oliva, 1999). Desde esta perspectiva, los más variados frentes didácticos de clase (debates, experiencias, analogías, problemas, explicaciones, etc.) pueden ser válidos para enriquecer el significado del alumno. Todos los modelos de enseñanza tienen cierto grado de validez y son, en cierta medida, complementarios (Aliberas, Gutiérrez e Izquierdo, 1989).

- Es posible fomentar el desarrollo cognitivo del alumno activando sus mecanismos de toma de conciencia a fin de hacer explícitos sus conocimientos implícitos. El proceso de conceptualización no es sólo ligar lo implícito con representaciones simbólicas; requiere de nuevas construcciones cognitivas (Piaget, 1976). Además, al hacerse representacional, el sujeto adquiere un mayor control del contenido cognitivo implícito (Karmiloff-Smith, 1994; Pozo y Gómez Crespo, 1998).

La anterior lista de implicaciones didácticas basadas en el «alumno como aprendiz» dependerá del modelo usado sobre el conocimiento del alumno. Así, considerando el modelo de equilibrio de Piaget (1978), se podría llegar a nuevas sugerencias didácticas, por ejemplo, considerando los procesos de abstracción reflexiva en la construcciones operacionales o los diferentes modos de salvar el conflicto cognitivo (Marín, 1997).

Por último, desde los modelos $A c C$ se afirma con frecuencia que «la educación científica del alumno se debe plantear en consonancia con el quehacer científico» (Gil et al., 
1999). Análogamente, desde la perspectiva del aprendiz se podría afirmar «cuanto más ajustados y precisos se hagan los diseños de enseñanza a los procesos de aprendizaje y desarrollo cognitivo que llevan al novato a convertirse en experto en ciencias, mayores serán las posibilidades para provocar esta transformación cognitiva». Tanto un argumento como el otro gozan de semejante evidencia lógica.

Ahora bien, aunque la formación fuertemente disciplinar del investigador en el ámbito de la didáctica de las ciencias ha inclinado la balanza a favor del «argumento disciplinar» (Marín et al., 1999), no faltan razones para equili-

\section{REFERENCIAS BIBLIOGRÁFICAS}

ALIBERAS, J., GUTIÉRREZ, R. e IZQUIERDO, M. (1989). Modelos de aprendizaje en la didáctica de las ciencias. Investigación en la Escuela, 9, pp. 17-24.

ARTIGUE, M. y DOUADY, R. (1986). La Didactique des Mathématiques en France. Revue Française de Pedagogie, 76, pp. 69-88.

AUSUBEL, D.P., NOVAK, J.D. y HANESIAN, H. (1986). Psicología educativa. Un punto de vista cognoscitivo. Méjico: Trillas.

BOTELLA, L. (1994). "Constructivismo y desarrollo del conocimiento: Procesos ortogenéticos de evolución de las teorías personales». Tesis doctoral no publicada.

BUNGE, M. (1981). La investigación científica. Barcelona: Ariel.

CAREY, S. (1991). Knowledge acquisition: Enrichment or conceptual change?, en Carey, S. y Gelman, R. The epigenesis of mind. Erlbaum: Hillsdale, Nueva Jersey.

CAREY, S. y SPELKE, E. (1994). Domain specific knowledge and conceptual change, en Hirschfeld, L. y Gelman, S. (eds.). Mapping the mind. Cambridge, MA: Cambridge University Press.

CASTILlA DEL PINO, C. (2000). Teoría de los sentimientos. Barcelona: Tusquets.

CHALMERS, A.F. (1984). ¿Qué es esa cosa llamada ciencia? Madrid: Siglo XXI.

CHI, M.T.H., SLOTTA, J.D. y LEEUW, N. (1994). From Things to processes: A theory of conceptual change for learning science concepts. Learning and Instruction, 4, pp. 27-43.

CLAXTON, G. (1987). Vivir y aprender. Madrid: Alianza Editorial.

CLAXTON, G. (1994). Educar mentes curiosas. El reto de la ciencia en la escuela. Madrid: Visor.

CLEMENT, J. (2000). Model based learning as a key research area for science education. International Journal of Science Education, 22(9), pp. 1041-1053. brarla tomando argumentos desde el alumno como aprendiz. Si la educación científica se entiende, más que como adquisición de un saber disciplinado, elaborado y formalizado, como un enriquecimiento del conocimiento del alumno para actuar y comprender mejor el medio, entonces un modelo para la enseñanza de las ciencias debe buscar concordancia, más que con el modo con que se construye el conocimiento de ciencias, con el modo de construir el alumno su propio conocimiento. No se debe confundir un mayor acuerdo entre enseñanza de ciencias y quehacer científico con una mayor comprensión del alumno de los contenidos.

DE CUDMANI, L.C., PESA, M.A., SALINAS, J. (2000). Hacia un modelo integrador para el aprendizaje de las ciencias. Enseñanza de las Ciencias, 18(1), pp. 3-13.

DE POSADA, J.M. (1996). Hacia una teoría sobre las ideas científicas de los alumnos: influencia del contexto. Enseñanza de las Ciencias, 14(3), pp. 303-314.

DELVAL, J. (1997). Tesis sobre el constructivismo, pp. 15-24, en Rodrigo, M.J. y Arnay, J. (comps.). La construcción del conocimiento escolar. Barcelona: Paidós.

DI SESSA, A.A. y SHERIN, B.L. (1998). What changes in conceptual change. International Journal of Science Education, 20(10), pp. 1155-1191.

DRIVER, R. (1983). The pupil as scientist. Milton Keynes, UK: Open University Press.

DRIVER, R. (1986). Psicología cognoscitiva y esquemas conceptuales de los alumnos. Enseñanza de las Ciencias, 4(1), pp. 3-15.

DUIT, R. (1999). Conceptual change approaches in Science Education, pp. 263-282, en Schnotz, W. Vosniadou, S. y Carretero, M. (eds.). New perspectives on conceptual change. Londres: Elsevier.

DUSCHL, R.A. y GITOMER, D.H. (1991). Epistemological Perspectives on Conceptual Change: Implications for Educational Practice. Journal of Research in Science Teaching, 28(9), pp. 839-858.

FERNÁNDEZ, I., GIL, D. VILCHES, A., VALDÉS, P., CACHAPUZ, A., PRAIA, J. y SALINAS, J. (2002). La superación de las visiones deformadas de la ciencia y la tecnología: un requisito esencial para la renovación de la educación científica. II Congreso Internacional de Didáctica de las Ciencias: La enseñanza de las ciencias en el siglo XXI. La Habana, Cuba (11-15 de febrero de 2002). IPLAC. Edición en CD preparada por OEI. 
GIERE, R. (1992). Cognitive Models of Science. Minneapolis: University of Minnesota Press.

GIL, D. (1993). Contribución de la historia y de la filosofía de las ciencias al desarrollo de un modelo de enseñanzaaprendizaje como investigación. Enseñanza de las Ciencias, 11(2), pp. 197-212.

GIL, D., CARRASCOSA, J., DUMAS-CARRÉ, A., FURIÓ, C., GALLEGO, R. GENÉ, A., GONZÁLEZ, E., GUISASOLA, J., MARTÍNEZ-TORREGROSA, J., PESSOA DE CARVALHO, A.M., SALINAS, J., TRICÁRICO, H. y VALDES, P. (1999). ¿Puede hablarse de consenso constructivista en la educación científica? Enseñanza de las Ciencias, 17(3), pp. 503-512.

GILBERT, J.K. y SWIFT, D.J. (1985). Towards a lakatosian analysis of the piagetian and alternative conceptions research programs. Science Education, 69(5), pp. 681-696.

GOBERT, J.D. (2000). Introduction to model-based teaching and learning in science education, 22(9), pp. 891-894.

GRECA, I.M. y MOREIRA, M.A. (1998). Modelos mentales y aprendizaje de física en electricidad y magnetismo. Enseñanza de las Ciencias, 16(2), pp. 289-303.

HOLTON, G. (1972). Introducción a los conceptos y teorías de las ciencias físicas. Barcelona: Reverté. (Ed. orig. 1952. Massachusetts: Addisson-Wesley.)

INHELDER, B. y PIAGET, J. (1972). De la lógica del niño a la lógica del adolescente. Buenos Aires: Paidós. (Ed. orig. 1955. París: Presses Universitaires de France.)

IZQUIERDO, M. (2000). Fundamentos epistemológicos, pp. 3564, en Perales, F.J. y Cañal, P. (eds.). Didáctica de las Ciencias Experimentales. Alcoy: Marfil.

JIMÉNEZ ALEIXANDRE, P. y SANMARTÍ, N. (1997). ¿Qué ciencia enseñar?: objetivos y contenidos de la educación secundaria, pp. 17-46, en Carmen, del L. La enseñanza y el aprendizaje de las Ciencias de la Naturaleza en la educación secundaria. Barcelona: ICE-Horsori.

JIMÉNEZ ALEIXANDRE, M.P. (2000). Modelos didácticos, pp. 165-186, en Perales, F.J. y Cañal, P. (eds.). Didáctica de las ciencias experimentales. Alcoy: Marfil.

KARMILOFF-SMITH, A. (1994). Más allá de la modularidad. Madrid: Alianza Editorial.

KELLY, G.A. (1955). The psychology of personal constructs. Londres: Routledge.

KUHN, T.S. (1975). La estructura de las revoluciones cientificas. Madrid: Fondo de Cultura Económica.

LAKATOS, I. (1974). Historia de la ciencia y sus reconstrucciones racionales. Madrid: Tecnos.

LAKATOS, I. (1983). La metodología de los programas de investigación cientifica. Madrid: Alianza Universitaria.

LAWSON, A.E. (1993). Deductive reasoning, brain maduration and science concept acquisition: Are they linked? Journal of Research in Science Teaching, 30(9), pp. 1029-1051.

LAWSON, A.E. (1994). Uso de los ciclos de aprendizaje para la enseñanza de destrezas de razonamiento científico y de sistemas conceptuales. Enseñanza de las Ciencias, 12(2), pp. 165-187.
LEACH, J. (1999). Students' undertanding of the co-ordination of theory and evidence in science. International Journal of Science Education, 21(8), pp. 789-806.

LUFFIEGO, M., BASTIDA, M.F., RAMOS, F. y SOTO, J. (1994). Sistemic model of conceptual evolution. International Journal of Science Education, 16(3), pp. 305-313.

LUFFIEGO, M. (2001) Reconstruyendo el constructivismo: hacia un modelo evolucionista del aprendizaje de conceptos. Enseñanza de las Ciencias, 19(3), pp. 377-392.

MARÍN, N. (1986). Estudios sobre las SRT de Shaver, M. VII Encuentros de Didáctica de la Física y Química. Jaén.

MARÍN, N. (1995). Metodología para obtener información del alumno de interés didáctico. Almería: Servicio de Publicaciones de la Universidad de Almería.

MARÍN, N. (1997). Fundamentos de didáctica de las ciencias experimentales. Almería: Servicio de Publicaciones de la Universidad de Almería.

MARÍN, N. (1999). Delimitando el campo de aplicación del cambio conceptual. Enseñanza de las Ciencias, 17(1), pp. 79-92.

MARÍN, N., SOLANO, I. y JIMÉNEZ GÓMEZ, E. (1999). Tirando del hilo de la madeja constructivista. Enseñanza de las Ciencias, 17(3), p. 479-492.

MARÍN, N. y BENARROCH, A. (2000). Precisiones sobre el constructivismo e implicaciones para la educación. Paideia. Revista de Educación, 28. Chile: Universidad de Concepción.

MARÍN, N., SOLANO, I. y JIMÉNEZ GÓMEZ, E. (2001). Characteristics of the methodology used to describe students' conceptions. International Journal of Science Education, 23(7), pp. 663-690.

MARINA, J.A. (1998). La selva del lenguaje. Barcelona: Anagrama.

MEC (1989). Diseño curricular base. Educación primaria (I y II). Madrid: MEC.

OLIVA, J.M. (1996). Estudios sobre consistencia de las ideas de los alumnos en ciencias. Enseñanza de las Ciencias, 14(1), pp. 87-92.

OLIVA, J.M. (1999). Algunas reflexiones sobre las concepciones alternativas y el cambio conceptual. Enseñanza de las Ciencias, 17(1), pp. 93-108.

OSBORNE, J.F. y SIMON, S. (1996). Primary Science: past and future directions. Studies in Sciencie Education, 26, pp. 99-147.

PIAGET, J. (1974). El estructuralismo. Barcelona: Oikos-Tau.

PIAGET, J. (1976). La toma de conciencia. Madrid: Morata. (Ed. orig. 1974. París: Presses Universitaires de France.)

PIAGET, J. (1977). Epistemología genética. Argentina: Solpin. (Ed. orig. 1970. L'epistemologie génetique. París: Presses Universitaires de France.)

PIAGET, J. (1978). La equilibración de las estructuras cognitivas, Problema central del desarrollo. Madrid: Siglo XXI.

PIAGET, J. (1980). Psicología y pedagogía. Barcelona: Ariel. (Ed. orig. 1969. París: Gonthier.) 
PIAGET, J. y GARCÍA, R. (1982). Psicogénesis e historia de la ciencia. México: Siglo XXI.

PINTRICH, P.R. (1999). Motivacional Beliefs as Resources for and Constraints on Conceptual Change, pp. 33-50, en Schnotz, W., Vosniadou, S. y Carretero, M. (eds.). New perspectives on conceptual change. Londres: Elsevier.

POSNER, G.J., STRIKE, K.A., HEWSON, P.W. y GERTZOG, W.A. (1982). Accomodation of a Scientific Conception: Toward a Theory of conceptual change. Science Education, 66(2), pp. 211-227.

POZO, J.I. (1989). Teorías cognitivas del aprendizaje. Madrid: Morata.

POZO, J.I. (1996). No es oro todo lo que reluce ni se construye (igual) todo lo que se aprende: contra el reduccionismo constructivista. Anuario de Psicología, 69, pp. 127-139.

POZO, J.I. (1999). Sobre las relaciones entre el conocimiento cotidiano de los alumnos y el conocimiento científico: del cambio conceptual a la integración jerárquica. Enseñanza de las Ciencias, núm. extra, pp. 15-31.

POZO, J.I., GÓMEZ CRESPO, M.A., LIMÓN, M. y SERRANO SANZ, A. (1991). Procesos cognitivos en la comprensión de la ciencia: las ideas de los adolescentes sobre la química. Madrid: CIDE (MEC).

POZO, J.I. y POSTIGO, Y. (1994). La solución de problemas como contenido procedimental de la educación obligatoria, pp. 180-213, en Pozo et al. La solución de problemas. Madrid: Aula XXI - Santillana.

POZO, J.I. y GÓMEZ CRESPO, M.A. (1998). Aprender y enseñar ciencia. Madrid: Morata.

POZO, J.I. y SCHEUER, N. (1999). Las concepciones sobre el aprendizaje como teorías implícitas, pp. 87-108, en Pozo, J.I. y Monereo, C. El aprendizaje estratégico. Madrid: Aula XXISantillana.

REIF, F. y LARKIN, J.H. (1991). Cognition in scientific and everyday domains: Comparison and learning implications. Journal of Research in Science Teaching, 28(9), pp. 733-760.

RIVIÈRE, A. (1990). La teoría cognitiva social del aprendizaje: implicaciones educativas, pp. 69-80, en Coll, C., Palacios, J. y Marchesi, A. Desarrollo psicológico y Educación, II. Madrid: Alianza Editorial.

RODRIGO, M.J. (1997). Del escenario sociocultural al constructivismo episódico: un viaje al conocimiento escolar de la mano de las teorías implícitas, pp. 177-194, en Rodrigo, M.J. y Arnay, J. (comps.). La construcción del conocimiento escolar. Barcelona: Paidós.

RODRIGO, M.J., RODRÍGUEZ, A. y MARRERO, J. (1993). Las teorías implícitas. Una aproximación al conocimiento cotidiano. Madrid: Visor.

RODRIGO, M.J. y CORREA, N. (1999). Teorías implícitas, modelos mentales y cambio educativo, pp. 75-86, en Pozo, J.I. y Monereo, C. El aprendizaje estratégico. Madrid: Aula XXI - Santillana.
RUMELHART, D.E. y ORTONY, A. (1982). The representation of knowledge in memory. Infancia y aprendizaje, 20, pp. 115-158.

SCHNOTZ, W., VOSNIADOU, S. y CARRETERO, M. (1999). New trends on conceptual change. Londres: Elsevier.

SHAYER, M. y ADEY, P. (1984). La ciencia de enseñar ciencia, desarrollo cognoscitivo y exigencias del curriculum. Madrid: Narcea.

SHAYER, M. y ADEY, P.S. (1993). Accelerating the development of formal thinking in middle and high school students IV: three years after a two years intervention. Journal of Research in Science Teaching, 30(4), pp. 351-366.

SOLOMON, J. (1994). The rise and fall of constructivism. Studies in Science Education, 23, pp. 1-19.

STEINBERG, M.S., BROWN, D.E. y CLEMENT, J. (1990). Genius is not immune to persistent misconceptions: conceptual difficulties impeding Isaac Newton and contemporary physics students. International Journal of Science Education, 12(3), pp. 265-273.

THAGARD, P.R. (1992). Conceptual revolutions. Princeton, N.J.: Princeton University Press.

TOULMIN, S. (1972). Human understanding Vol I. The collective usage and evolution of concepts. Pricenton: Pricenton University Press. Trad. cast. (1977). La comprensión humana (I). El uso colectivo y la evolución de los conceptos. Madrid: Alianza Universitaria.

VALDÉS, P., GIL, D., VILCHES, A. y MARTÍNEZTORREGROSA，J. (2002). ¿Qué entendemos por constructivismo en didáctica de las ciencias? II Congreso Internacional de Didáctica de las Ciencias: La enseñanza de las ciencias en el siglo XXI. La Habana. Cuba (11-15 de febrero de 2002). IPLAC. Edición en CD preparada por OEI.

VÁZQUEZ, A., ACEVEDO, J.A., MANASSERO, M.A. y ACEVEDO, P. (2001). Cuatro paradigmas básicos sobre la naturaleza de la ciencia. Argumentos de Razón Técnica, 4, pp. 135-176.

VILLANI, A. y ORQUIZA DE CARVALHO, L. (1995). Conflictos cognitivos, experimentos cualitativos y actividades didácticas. Enseñanza de las Ciencias, 13(3), pp. 279-294.

VOSNIADOU, S. (1994). Capturing and modelling the process of conceptual change. Learning and Instruction, 4(1), pp. 45-69.

VOSNIADOU, S. (1999). Conceptual change research: state of the art future directions, pp. 3-14, en W. Schnotz, Vosniadou, S. y Carretero, M. (eds.). New perspectives on conceptual change. Londres: Elsevier.

YANG, W.G. (1999). An Analysis of «Pupil as Scientist» Analogies. Ponencia presentada en el V International History, Philosophy and Science Teaching Conference: Science as Culture. Bicentenary of the Invention of the Battery by Alessandro Volta. Lago Como. Italia. (15-19 de septiembre). 